\title{
Caracterización epidemiológica de mordeduras en personas, según registro de atención de urgencia. Provincia de Los Andes, Chile
}

\author{
Vania Villagra, Dante Cáceres, Sergio Alvarado, Elizabeth Salinas, \\ M. Loreto Caldera, Erick Lucero, Paola Viviani y Marisa Torres
}

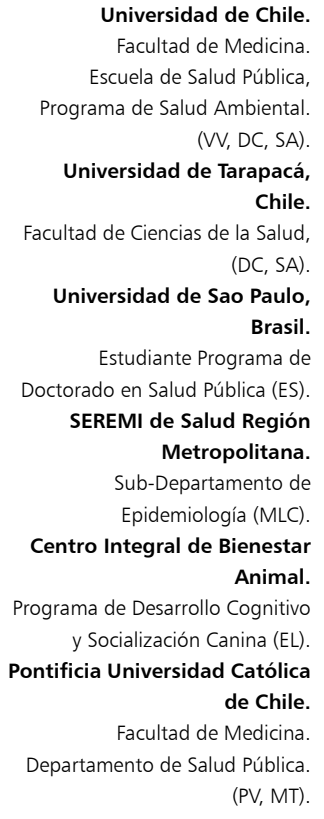

Universidad de Chile.

Facultad de Medicina. Escuela de Salud Pública Programa de Salud Ambiental. $(\mathrm{VV}, \mathrm{DC}, \mathrm{SA})$ Universidad de Tarapacá, Chile. Facultad de Ciencias de la Salud, (DC, SA).

Universidad de Sao Paulo, Brasil.

Estudiante Programa de Doctorado en Salud Pública (ES). SEREMI de Salud Región Metropolitana.

Sub-Departamento de Epidemiología (MLC) Centro Integral de Bienestar Animal. Programa de Desarrollo Cognitivo y Socialización Canina (EL) Pontificia Universidad Católica de Chile.

Facultad de Medicina. Departamento de Salud Pública. (PV, MT).

Los autores declaran no tener conflictos de interés. Fuente de financiamiento: Propio.

Recibido: 11 de agosto de 2016 Aceptado: 18 de mayo de 2017

Correspondencia a: Marisa Torres Hidalgo marisa@med.puc.cl

\section{Introducción}

L as mordeduras por animales a personas, constituyen un grave problema de salud pública mundial y nacional. Afectan de manera transversal a niños y adultos de todas las edades y nivel socio económico. Las consecuencias de las mordeduras para la salud humana dependen de factores relacionados con las características del animal mordedor (especie, tamaño y estado de salud) y de la persona mordida (edad, tamaño, estado de salud $\mathrm{y}$ acceso para atención). Las mordeduras originan gran cantidad de heridas que requieren atención médica y o quirúrgica más terapia anti-rábica preventiva ${ }^{1-4,19,32}$.

Estos accidentes generan importantes gastos económicos al sistema de salud y a la familia afectada. Según antecedentes de la Organización Mundial de la Salud (OMS), cada año más de 7 millones de personas en el mundo son mordidas por perros ${ }^{32}$. En Chile entre los años 2003 y 2012, se reportaron 327.150 personas mordidas (PM) con una tasa de 196/100.000 hbts. Según el Servicio de Salud Aconcagua, entre los años 2003 y 2012 se registraron 3.343 personas mordidas y una tasa anual promedio de 146 por 100.000 hbts. ${ }^{4-9,32}$.

$\mathrm{La}$ agresividad entre caninos corresponde a una forma de comunicación cuya función es regular las relaciones sociales dentro del grupo; éstas se realizan a través de posturas de sumisión o dominancia. Se considera que las agresiones de caninos contra un ser humano se producen por una mala comunicación entre éste y su mascota, ya sea porque la persona no percibe las señales de alerta corporales (cola rígida) o faciales (mirada fija, exponer dientes) que emite el animal, cuando reacciona protegiendo algún recurso importante para él (territorio, recursos alimenticios) o como respuesta ante el miedo. En cambio, la agresividad en gatos es una respuesta del animal a la inestabilidad que percibe en el territorio o por invasión de éste, y o a la falta de socialización del gato con la especie humana a temprana edad. Entre las situaciones que generan inestabilidad en el territorio del gato se encuentran, cambios de casa, visitas (niños), ruidos, presencia 
de otro animal. La importancia para la salud humana de las agresiones por gatos está relacionada, entre otras, al riesgo de adquirir la enfermedad por rasguño de gato (bartonelosis), cuya presentación es más grave en niños, adultos mayores y personas inmunocomprometidas ${ }^{10,11}$.

Desde el punto de vista de la vigilancia epidemiológica y del control del problema, Chile no cuenta con normativa para manejo de las poblaciones de animales de compañía, en lo referido a obligaciones de los propietarios, resguardar la seguridad de las personas y regular la responsabilidad por daños. No obstante, existe normativa sanitaria para prevención y control de enfermedades específicas de mordeduras como tétanos y rabia; el Reglamento de Prevención y Control de Rabia en el hombre y los animales DS N ${ }^{\circ} 1$ del 2013 y la norma técnica de vacunación anti-rábica en humanos, indican el procedimiento a seguir en caso de personas mordidas ${ }^{13-16}$. Los establecimientos de salud que atienden personas mordidas deben hacer una exhaustiva entrevista al paciente con el fin de determinar la conducta médica a seguir y notificar el caso a la Autoridad Sanitaria para completar la vigilancia de rabia "Denuncia de accidentes por mordeduras ${ }^{32 "}$. De esta manera, las notificaciones de mordeduras de los centros de salud de Chile y del mundo, se convierten en la principal fuente de información para conocer las implicancias epidemiológicas de este tipo de accidentes $^{14,15,17,18}$.

La Provincia de Los Andes se ubica en el sector este de la Región de Valparaíso y está constituida por cuatro comunas: Los Andes, San Esteban, Calle Larga y Rinconada, siendo la ciudad de Los Andes su capital provincial. Cuenta con una superficie de $3.054 \mathrm{~km}^{2}$, y posee una población estimada al 30 de junio de 2015 de 111.322 hbts. $^{21}$ (Figura 1).

En Chile la población total canina fluctúa entre 2,6 y 3 millones de perros y $75 \%$ de estos animales, pese a poseer un propietario, deambulan sin sujeción ni control alguno por las calles ${ }^{33}$. Se estima que hasta abril de 2014 había 1.436 perros sin dueños, existiendo una relación de un perro sin dueño por cada 45 hbts. en la comuna de Rinconada ${ }^{34}$. En el resto de las comunas no se ha realizado algún estudio para determinar las características demográficas de las poblaciones caninas y felinas. Sin embargo, al realizar una visita diagnóstica y al consultar a funcionarios municipales, se obtuvo evidencias sobre la sensación de inseguridad de las personas al transitar por

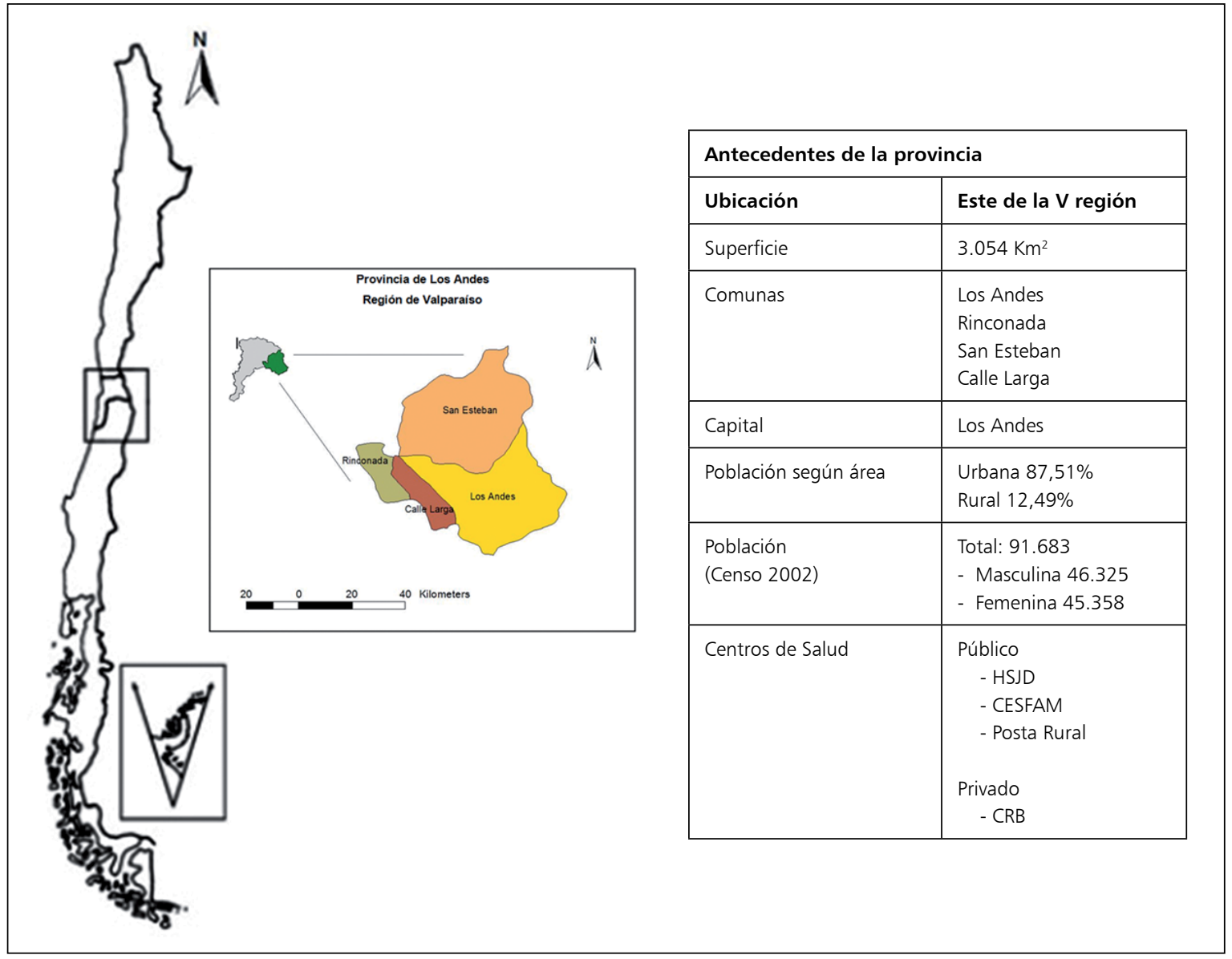

Rev Chilena Infectol 2017; 34 (3): 212-220
Figura 1. Antecedentes de la Provincia de Los Andes. Fuente: Elaboración propia según información INE censo 2002, Servicio de Salud Aconcagua ${ }^{20-22}$. 
distintos sectores de las comunas debido a la presencia de perros deambulando libremente por las calles; no existen antecedentes sobre algún trabajo similar realizado en la provincia de Los Andes.

El presente estudio, tuvo como objetivo caracterizar epidemiológicamente los accidentes por mordeduras de animales en humanos ocurridos en la Provincia de Los Andes en el período 2005-2007.

\section{Materiales y Método}

\section{Diseño y población en estudio}

Se realizó un estudio epidemiológico descriptivo, retrospectivo, en el período enero de 2005 a diciembre de 2007, en la Provincia de Los Andes. Se analizaron las estadísticas sobre personas mordidas (n: 2.360) atendidas, registradas en las bases de datos secundarias del Servicio de Urgencia del HSJD y CRB (pacientes por ingreso y diagnóstico mordeduras) y notificaciones de accidente por mordedura, disponibles en la Oficina de Zoonosis, SEREMI de Salud Aconcagua ${ }^{14}$.

Se analizaron los siguientes factores: de la persona mordida (antecedentes socio demográficos, como género, edad, distribución geográfica); del accidente por mordedura se analizó temporalidad (año, mes y estación); del animal mordedor (especie, ubicación geográfica, condición de propiedad, denuncia de animal mordedor) y de la mordedura (número, ubicación, complicaciones).

\section{Análisis estadístico}

El muestreo fue no probabilístico por conveniencia. Se depuraron las bases de datos (retiro de casos repetidos por la misma mordedura). Se realizó análisis exploratorio de datos calculando frecuencia y porcentaje en cada variable. Se usó el programa SPSS v17. Las tasas fueron calculadas según proyección de Censo 2002.

\section{Aspectos éticos}

En este estudio se usaron fuentes secundarias de información, que contienen datos sensibles de los pacientes mordidos en el período de estudio. El compromiso de los autores fue acoger los principios fundamentales de la investigación con seres humanos, el respeto por las personas (dignidad humana y autonomía), la beneficencia y la justicia. En este contexto se ha garantizado el resguardo de la confidencialidad de la información personal y datos sensibles en salud sobre personas mordidas, impidiendo cualquier tipo de daño por faltas al resguardo del debido secreto o privacidad que puede derivar en problemas familiares, laborales, como también estigma social.

Además, se garantizó el uso responsable de los datos otorgados que contienen la información personal de los pacientes atendidos por mordeduras. En la difusión de resultados se mantiene el anonimato. Desde el punto de vista de la ética de prevención, la realización de este estudio aporta al conocimiento de la magnitud y consecuencias de los accidentes por mordeduras, lo que permitirá sugerir medidas de prevención en el entorno social y ambiental estudiado y otros similares, esto en beneficio de las comunidades locales ${ }^{29,30}$.

\section{Limitaciones}

Falta de acceso a información (2008-2016), considerando la Ley $\mathrm{N}^{\circ} 20.285$ sobre acceso a información pública en vigencia desde el año 2012 no fue factible acceder a los datos sobre personas mordidas en los centros de salud ubicados en la Provincia de Los Andes.

- Registro en notificación de animal mordedor y bases de datos incompletas.

\section{Resultados}

\section{Distribución anual de personas mordidas (PM)}

Se observaron 2.360 PM atendidas en los centros de salud de la provincia durante el período, 2.186 personas tenían su residencia en Provincia de Los Andes. Durante el año 2006 se presentó la mayor frecuencia, con una tasa de $843 \mathrm{PM} / 100.000$ hbts y un promedio de 2,31 PM/día (Tabla 1).

Tabla 1. Distribución anual de PM atendidas en los servicios de urgencia. Provincia de Los Andes y con residencia en la Provincia de Los Andes (2005-2007)

Personas atendidas en los servicios de urgencia. Provincia de Los Andes

Año

2005

2006

2007

Total

\begin{abstract}
Total atendido
\end{abstract}
n

679

914

767

2.360

$\%$

28,7

38,7

32,5

100
Promedio*

1,8

2,5

2,1

2,1
Personas atendidas con residencia en la Provincia de Los Andes

\begin{tabular}{cccc}
\multicolumn{2}{c}{ Total de residentes atendidos } & Tasa** & Promedio*** $_{\mathbf{n}}^{*}$ \\
636 & 29,0 & 644 & 1,74 \\
842 & 38,5 & 843 & 2,31 \\
708 & 32,3 & 700 & 1,94 \\
2.186 & 100,0 & 729 & 1,99
\end{tabular}

*Promedio diario de personas mordidas atendidas en los servicios de urgencia de la Provincia. **Tasa anual por cada 100.000 habitantes. ***Promedio diario de personas mordidas con domicilio en la Provincia de Los Andes. PM: Personas Mordidas. 


\section{Distribución temporal}

Las mordeduras se presentaron durante todo el año, observándose la frecuencia máxima en diciembre. En cuanto a la presentación semanal, la mayor frecuencia estuvo el día domingo.

\section{Distribución según género}

Las mordeduras predominaron en los hombres y la mayor tasa se presentó el año 2006 (Tabla 2).

\section{Distribución según grupo etario y sexo}

Se encontraron personas mordidas en todos los grupos etarios. Los niños bajo 10 años presentaron la mayor frecuencia de mordeduras y la mayor tasa se observó en el grupo de 6 a 10 años (1.521/100.000 hbts) (Tabla 3). Al analizar las personas mordidas según edad y sexo, se observa que las mordeduras de hombres predominaron hasta los 30 años; desde los 31 años en adelante predominaron en las mujeres (Figura 2).

\section{Distribución del tipo de animal mordedor}

Se registraron diferentes especies de animal mordedor. El perro fue la especie que predominó (67\% de las mordeduras), le siguieron las arañas y los gatos (Tabla 4). Al analizar la relación entre especie y género de la persona mordida, se observó que las mordeduras de perro, araña, gato y ratón afectaron a hombres y mujeres, las de perros predominaron en hombres y las de araña, gato y ratón en las mujeres.

\section{Distribución temporal según especie}

Las mordeduras de perros, gatos y arañas ocurrieron durante todo el año. Las mordeduras de perros y gatos se

\begin{tabular}{lrr}
$\begin{array}{l}\text { Tabla 4. PM según animal mordedor. Provincia de Los } \\
\text { Andes (2005-2007) }\end{array}$ & Total \\
\hline Animal mordedor & $\mathbf{n}$ & $\%$ \\
\hline Perro & 1.584 & 67,1 \\
\hline Araña & 167 & 7,0 \\
\hline Gato & 93 & 3,9 \\
\hline Roedor & 15 & 0,6 \\
\hline Humano & 7 & 0,3 \\
Caballo & 4 & 0,1 \\
\hline Hamster & 3 & 0,1 \\
\hline Culebra & 2 & 0,0 \\
\hline Conejo & 1 & 0,0 \\
\hline NC & 484 & 20,5 \\
\hline Total & 2.360 & 100,0 \\
\hline
\end{tabular}

NC: No Consignado. PM: Personas Mordidas.

\begin{tabular}{ccccccc}
\multicolumn{2}{c}{ Tabla 2. Distribución de PM y tasas según sexo. Provincia de Los Andes (2005-2007) } \\
\hline Año & $\mathbf{n}$ & $\begin{array}{c}\text { Masculino } \\
\%\end{array}$ & Tasa* & $\mathbf{n}$ & $\begin{array}{c}\text { Femenino } \\
\%\end{array}$ & Tasa* $^{*}$ \\
2005 & 336 & 28,7 & 671 & 300 & 29,5 & 617 \\
2006 & 447 & 38,2 & 882 & 395 & 38,8 & 802 \\
2007 & 386 & 33,0 & 753 & 322 & 31,6 & 646 \\
\hline Total & 1.169 & 100,0 & 769 & 1.017 & 100,0 & 688 \\
\hline
\end{tabular}

*Tasa anual por cada 100.000 habitantes. Cálculo de tasas según PM con domicilio en la Provincia de Los Andes (n: 2.186). PM: Personas mordidas.

\begin{tabular}{|c|c|c|c|}
\hline \multirow[t]{2}{*}{ Grupo etario } & \multicolumn{2}{|c|}{ Total } & \multirow[t]{2}{*}{ Tasa* } \\
\hline & $\mathbf{n}$ & $\%$ & \\
\hline $0-5$ & 337 & 15,4 & 1.286 \\
\hline $6-10$ & 366 & 16,7 & 1.521 \\
\hline $11-15$ & 211 & 9,6 & 795 \\
\hline $16-20$ & 189 & 8,6 & 693 \\
\hline $21-30$ & 238 & 10,8 & 518 \\
\hline $31-40$ & 213 & 9,7 & 479 \\
\hline $41-50$ & 240 & 10,9 & 554 \\
\hline $51-60$ & 185 & 8,4 & 623 \\
\hline $61+$ & 205 & 9,3 & 636 \\
\hline NC & 2 & 0,0 & 0 \\
\hline Total & 2.186 & 100,0 & 729 \\
\hline
\end{tabular}

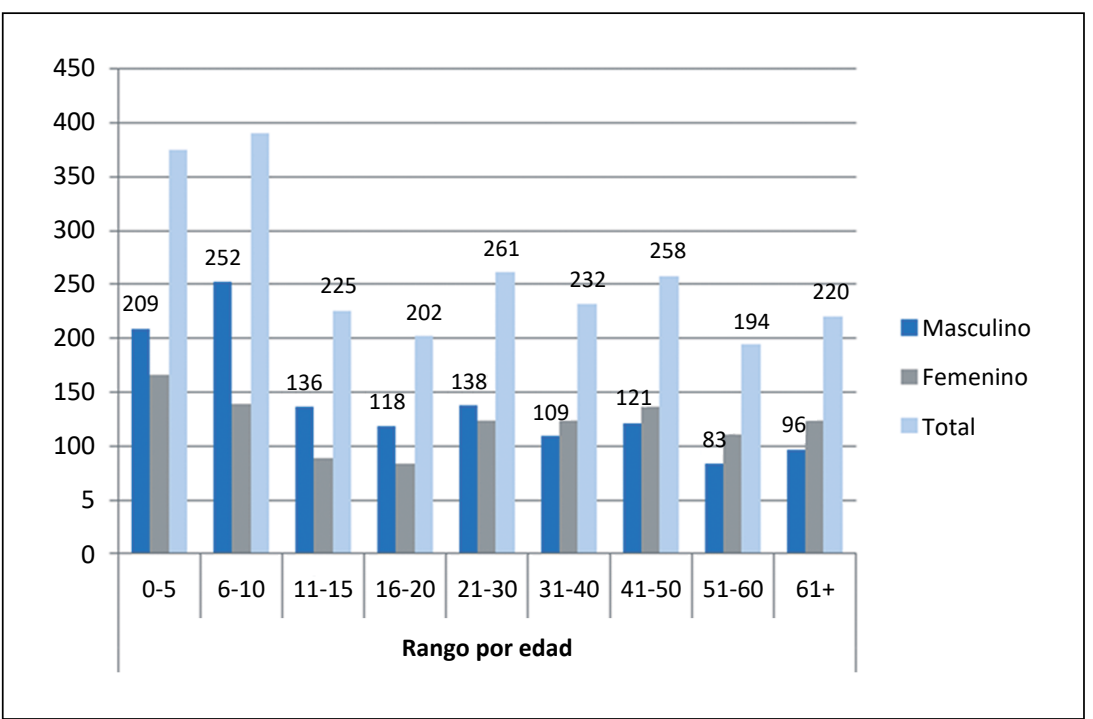

Figura 2. Distribución de PM según género y grupo etario. Provincia de Los Andes (2005-2007). PM: Personas mordidas. 
Figura 3. Distribución de mordeduras según especie y mes. Provincia de Los Andes (2005-2007).

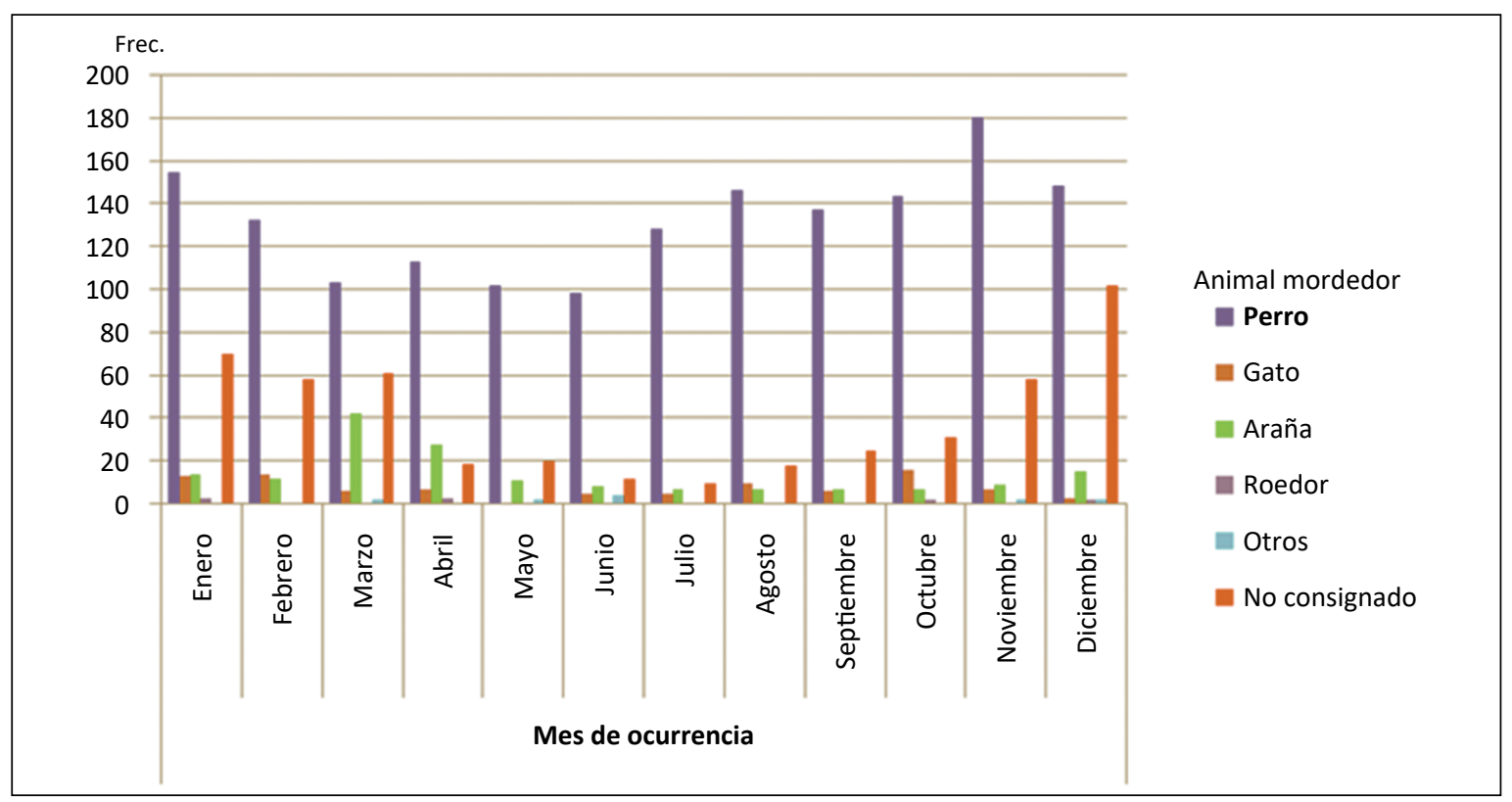

presentaron con mayor frecuencia entre octubre y marzo, las mordeduras por arañas se presentaron con mayor frecuencia durante marzo y abril. Los datos no consignados presentaron un aumento desde noviembre, alcanzando un máximo en diciembre para ir disminuyendo entre abril y junio (Figura 3).

\section{Distribución de características de las mordeduras}

En este estudio predominaron las mordeduras únicas en la región del tronco y extremidades (n: 1.023). Sin embargo, en 93,6\% (n: 2.209) de los casos hubo datos no consignados para caracterizar el número de mordeduras y casi 50\% (n: 1.171) de datos no consignados para la ubicación topográfica de la mordedura. En cuanto a las complicaciones, se observó mayor presentación de alergia no específica en ambos géneros $(28,7 \%$ ) (Tabla 5). En relación al género y ubicación de la mordedura, la mayoría de las mordeduras en ambos géneros afectó las extremidades inferiores (Figura 4). Es de destacar que las mordeduras localizadas en la cabeza afectaron en $50 \%$ (n: 83 ) a niños entre 0 y 5 años; esta ubicación física implica gran daño (Tabla 6).

\section{Distribución de complicaciones por mordeduras según especie y género}

Entre las complicaciones reiteradas, se encontró dolor por mordeduras de perro (n: 9), enfermedad por rasguño de gato (n: 17) y alergia no específica por mordedura de araña (n: 27); las complicaciones generadas por mordeduras de araña y gato afectaron más al género femenino ${ }^{32}$.

\section{Distribución espacial}

El 92,6\% (n: 2.186) de las PM y 32,8\% (n: 264) de los animales mordedores tenían su domicilio en la provincia de Los Andes. Para la variable domicilio del animal mordedor hubo $57 \%$ (n: 459) de datos no consignados.

Tabla 5. Distribución de mordeduras según ubicación topográfica específica. Provincia de Los Andes (2005-2007)

\begin{tabular}{lcc}
\hline Cabeza cuello & $\mathbf{n}$ & $\%$ \\
\hline Cabeza & 39 & 23,4 \\
\hline Mejilla & 35 & 21,0 \\
\hline Cara & 31 & 18,6 \\
\hline Labio & 25 & 15,0 \\
\hline Boca & 8 & 4,8 \\
\hline Párpado & 7 & 4,2 \\
\hline Nariz & 6 & 3,6 \\
\hline Cuello & 5 & 3,0 \\
\hline Ojo & 3 & 1,8 \\
Oído & 2 & 1,2 \\
\hline Ceja & 1 & 0,6 \\
\hline Cuero cabelludo & 1 & 0,6 \\
\hline Garganta & 1 & 0,6 \\
\hline Labio y mejilla & 1 & 0,6 \\
\hline Labio y lengua & 1 & 0,6 \\
\hline Total & 166 & 100,0 \\
\hline
\end{tabular}




\section{Distribución de mordeduras según comuna de residencia}

El 71,3\% de las personas mordidas tenía su residencia en la comuna de Los Andes, donde se presentó una tasa de mordeduras de 808/100.000 hbts. Le sigue la comuna de San Esteban con $14,91 \%$ de personas mordidas y una tasa de 680/100.000 hbts. En cuanto a la comuna de residencia del animal mordedor, fue posible obtener información a partir de 805 notificaciones de denuncia de animal mordedor. El 22\% de los animales mordedores tenían su domicilio en la comuna de Los Andes seguida por la comuna de San Esteban con 5,4\% (Tabla 7).

\section{Distribución de mordeduras según origen y condición de propiedad de animal mordedor}

Treinta y cinco por ciento (n: 287) de los animales eran conocidos por las víctimas. Más de 50\% (n: 483) de los datos sobre el domicilio del animal mordedor no fue consignado. Al analizar la condición de propiedad se obtuvo que $33,7 \%$ (n: 87 ) de las mordeduras fue ocasionada por un animal que pertenecía a la víctima.

\section{Diagrama de flujo de vigilancia animal mordedor}

La notificación de animal mordedor se aplicó a 47,6\% (n: 805) de las PM. Se realizaron 475 visitas y fue posible observar a 108 animales; de éstos, sólo nueve tenían vacuna anti-rábica vigente, representando $1,1 \%$ del total de formularios de notificaciones. De los animales no observados, $66 \%$ presentó direcciones incompletas o indeterminadas (Figura 5).

\section{Discusión}

En cuanto a la magnitud del fenómeno, se observaron diferencias entre las tasas reportadas por DEIS-MINSAL (167/100.000 habts) y SEREMI de Salud Valparaíso Oficina Aconcagua (320/100.000 hbts), existiendo una sub notificación estimada en $52,3 \%$. Es destacable la alta incidencia de mordeduras en niños de 6 a 10 años (tasa:1.521/100.000 hbts).

La distribución en el tiempo de las mordeduras presenta estacionalidad, existiendo incremento de la frecuencia entre los meses de octubre y marzo, siendo la mayor frecuencia en diciembre. Esto concuerda con otros estudios realizados en el país, en los cuales las mordeduras ocurren principalmente en los meses más calurosos del año. Los meses de invierno en la Provincia de Los Andes presentan temperaturas bajas que fluctúan entre 0 y $15{ }^{\circ} \mathrm{C}$, condición que lleva a las personas y animales a buscar lugares para protegerse del frío y tener menor interacción. Otros estudios señalan que por el frío en invierno las personas utilizan mayor cantidad de ropa, lo que los protege de los efectos de las mordeduras ${ }^{3,17-19,23}$.

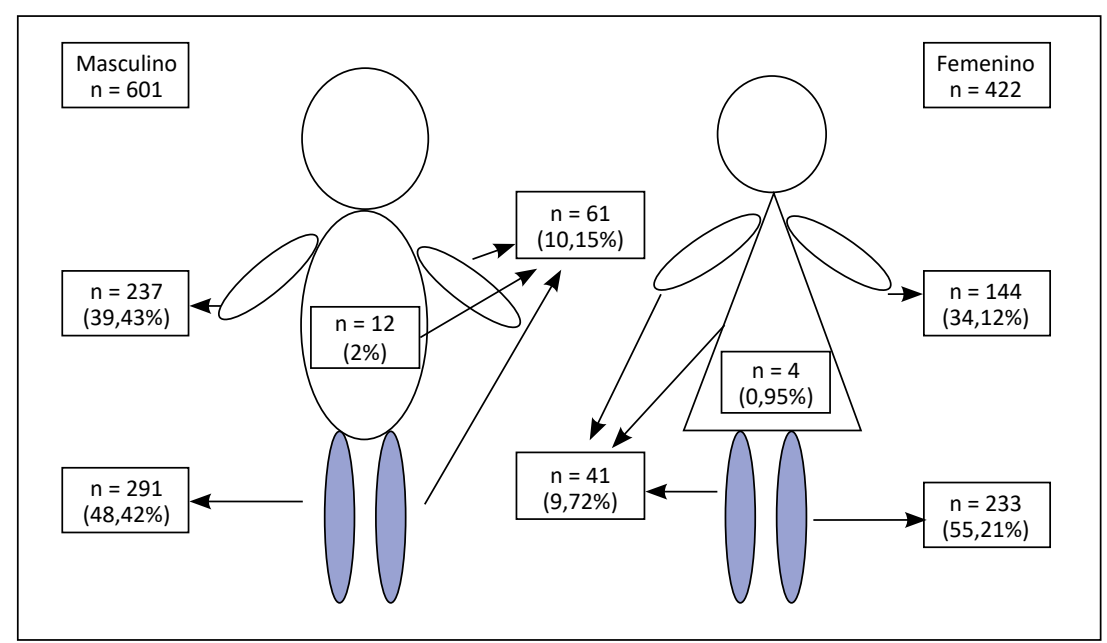

Figura 4. Frecuencia de lesiones ocasionadas en la región del tronco y extremidades según género. Provincia de Los Andes (2005-2007).

Tabla 6. Frecuencia y tasa comuna de residencia de PM y animal mordedor. Provincia de Los Andes (2005-2007)

\begin{tabular}{|c|c|c|c|c|c|}
\hline \multirow[t]{2}{*}{ Comuna } & \multicolumn{3}{|c|}{ Persona mordida } & \multicolumn{2}{|c|}{ Animal mordedor } \\
\hline & $\mathbf{n}$ & $\%$ & Tasa* & $\mathbf{n}$ & $\%$ \\
\hline Los Andes & 1.558 & 71,2 & 808 & 177 & 21,9 \\
\hline Rinconada & 86 & 3,9 & 364 & 20 & 2,4 \\
\hline San Esteban & 326 & 14,9 & 680 & 44 & 5,4 \\
\hline Calle Larga & 216 & 9,8 & 609 & 23 & 2,8 \\
\hline San Felipe & - & - & - & 3 & 0,3 \\
\hline Chacabuco & - & - & - & 1 & 0,1 \\
\hline Ignora domicilio & - & - & - & 78 & 9,6 \\
\hline No consignado & - & - & - & 459 & 57,0 \\
\hline Total & 2.186 & 100,0 & 787 & 805 & 100,0 \\
\hline
\end{tabular}

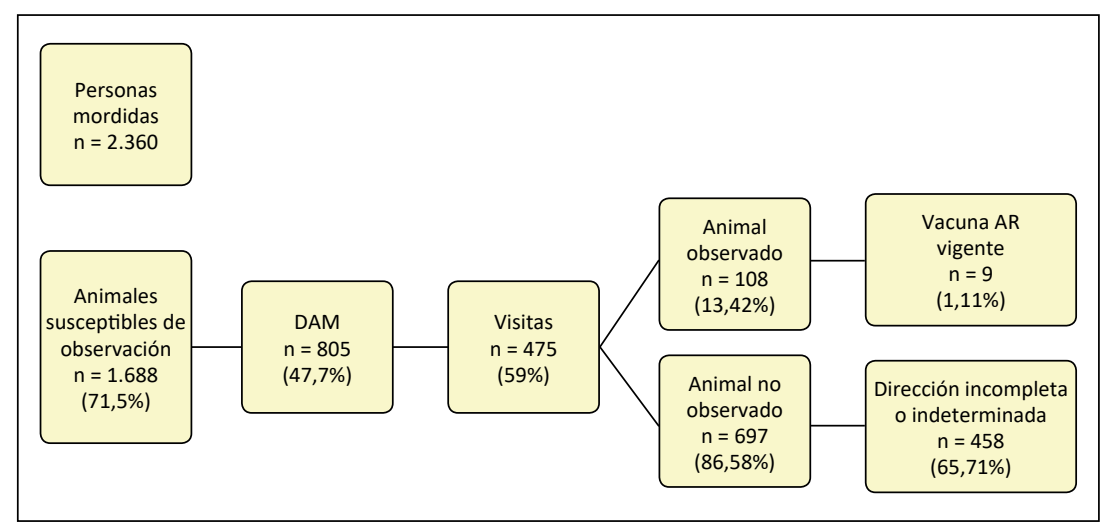

Figura 5. Diagrama de flujo: Observación del animal mordedor realizado por equipos de SEREMI Salud Aconcagua (art. 14. Reglamento de prevención y control de la Rabia en el hombre y animales). 
En general los estudios reportados en la literatura médica no registran las mordeduras de arañas.

La ubicación de mordeduras más frecuente estuvo en extremidades inferiores en las víctimas sobre 15 años de edad, lo que indica, desde el punto de vista del comportamiento, que el animal sintió invasión de territorio y la persona mordida utilizó sus piernas para defenderse o huir $^{3,7,10,11,19,23}$. La frecuencia de mordeduras de perros, gatos y roedores es similar a la de otros estudios realizados en Chile y el extranjero ${ }^{1,3,4,7,17-19}$. Gómez, Méndez y Kandaa señalan que las mordeduras ocurren en el entorno familiar, situación que sólo se presentó en la comuna de San Esteban ${ }^{25-28}$. Debido a que los animales propios y conocidos causaron la mayor frecuencia de mordeduras, es de importancia valorar las características y el tipo de mascotas que cada familia decide adquirir y a la estrategia de educación que debe orientar el médico veterinario tratante para proteger la salud animal y humana.

En la Provincia de Los Andes se observó que 52,3\% de los pacientes mordidos no tenían la notificación de denuncia del animal mordedor, estimándose que el factor principal de sub notificación sería el incumplimiento del Art 24 del "Reglamento de prevención de la rabia en el hombre y los animales", lo que amerita un estudio de proceso. Además, es importante mencionar que en 484 $(20,5 \%)$ casos el animal mordedor no fue consignado. Esta situación es relevante debido a la condición epidemiológica respecto de la rabia en nuestro país. Es sabido que los murciélagos insectívoros constituyen el reservorio de rabia en Chile. Tadarida brasiliensis es la especie más frecuente en el sector urbano por lo que constituye un riesgo potencial para la presentación de casos de rabia en animales domésticos y en humanos ${ }^{31}$.

Como se señaló anteriormente, una de las limitaciones del estudio fue el no tener acceso a información desde los centros de salud debido a la ley de protección de los pacientes, por lo que no se contó con información de las personas mordidas consultantes de los centros de salud en el período 2008-2016. La oficina de zoonosis, de la SEREMI de Salud Aconcagua, puso a disposición las cifras de personas mordidas según las notificaciones emitidas desde los centros de salud ubicados en la Provincia de Los Andes, en el trienio 2013-2015, (2013 n: 390; 2014 n: 690; $2015 \mathrm{n}: 610)^{35}$. Con estos datos se puede verificar que el problema sigue siendo relevante, y que sería de interés evaluar la magnitud de la sub-notificación en este período.

\section{Conclusiones}

Las mordeduras constituyen un importante problema de Salud Pública en la provincia, ya que la tasa fue más alta que el promedio del país. Dentro de la provincia, la mayor tasa se observó en las comunas de Los Andes y
San Esteban. De las cuatro comunas analizadas, sólo en la comuna de San Esteban hubo concordancia entre el domicilio del animal mordedor y la persona mordida.

Las mordeduras más frecuentes ocurrieron en primavera y verano, siendo el grupo más afectado en magnitud e impacto clínico los niños bajo 10 años. Las complicaciones más frecuentes fueron la alergia no específica, el efecto tóxico por mordedura de araña y la enfermedad por rasguño de gato. De los animales conocidos, los propios (perros y gatos) causaron el mayor porcentaje de mordeduras. En el contexto de la vigilancia epidemiológica de las mordeduras llamó la atención el déficit en el registro (incompleto y sub-registro).

\section{Sugerencias}

Se sugiere el uso de plataformas computacionales para notificar en línea, lo que facilitaría el proceso y permitiría monitorizar el registro. El cambio de nombre del protocolo de registro de "Denuncia" a "Notificación obligatoria de personas mordidas", podría mejorar el registro, ya que la denuncia es asociada a aspectos punitivos por la comunidad local.

En futuros estudios sería importante incorporar aspectos relacionados con la circunstancia en que ocurrió la mordedura y el comportamiento del animal (aspectos etológicos). Es de relevancia fomentar proyectos educativos para prevención de mordeduras en establecimientos educacionales, organizaciones comunitarias y ONGs.

Agradecimientos. Al equipo de la Oficina Provincial Aconcagua SEREMI Salud Región de Valparaíso: Germán Bachler, Iván Wainnright y Sra. Lilia Fuentes, al equipo del Hospital San Juan de Dios: Rodrigo Infante y Sres. Graciela Zelaya y de la Clínica Río Blanco: Vilma Olave por permitir acceso y colaborar en los procesos para concretar este trabajo. Al equipo de la I. Municipalidad de Los Andes: Sr. Miguel González, Sra. Mariana González. Al equipo de la I. Municipalidad de Rinconada: Srta. Gilian Arancibia, Sra. Milanda Carvajal y Sr. Miguel Salinas, al Sr. Manuel Ibaceta de I. Municipalidad de San Esteban y al Sr. César Suazo de I. Municipalidad de Calle Larga: por disponer gentilmente de su tiempo y aportar información valiosa de cada comuna.

\section{Resumen}

Introducción: Las mordeduras constituyen un problema de salud pública mundial. Objetivo: Caracterizar epidemiológicamente las mordeduras por animales ocurridos en la provincia de Los Andes (2005-2007). Método: Estudio epidemiológico descriptivo retrospectivo. Variables estudiadas: Persona mordida (PM), accidente por mordedura, animal mordedor y mordedura. No fue factible obtener información más actualizada por ley de 
protección del paciente. Resultados: Se atendieron 2.360 PM en los Servicios de Urgencia del Hospital San Juan de Dios y Clínica Río Blanco. Se registró una tasa anual promedio de $729 \mathrm{PM} / 100.000$ hbts. y promedio diario 1,99 . El género más afectado fue el masculino $(53,5 \%)$, y el grupo etario de 6 a 10 años (tasa:1.521/100.000). La ubicación topográfica más frecuente fue extremidad inferior, excepto en niños bajo 5 años de edad en quienes predominó cabeza-cuello. El animal mordedor según frecuencia fue: perro $(67,1 \%)$ araña $(7,1 \%)$ y gato $(3,9 \%)$. La propiedad del animal fue $35,6 \%$ conocida y $30,7 \%$ propios. Las complicaciones más frecuentes fueron alergia no específica, efecto tóxico por mordedura de arañas y entre las infecciones destaca la enfermedad por rasguño de gato. La notificación de animal mordedor se aplicó a 47,6\% de las PM; 92,8\% de la información contenida estaba incompleta. Conclusiones: Las mordeduras en la Provincia de Los Andes presentaron tasas mayores que el promedio país ( 729 versus $188 / 100.000$ ), destacando la mayor magnitud en niños de 6 a10 años. En la provincia no se han realizado programas de control de mordeduras o estudios actualizados.

\section{Referencias biliográficas}

1.- Palacio J, León M, García-Belenguer S. Aspectos epidemiológicos de las mordeduras caninas. Gac. Sanit. 2005; 19 (1): 50-8. [Fecha de acceso 12 de marzo de 2008]. Disponible en: http://www.scielosp.org/pdf/gs/ v19n1/revision.pdf

2.- Besada A, Huiaier F, Florentino J. Los niños y los perros ¿Cómo reducir los riesgos y maximizar los beneficios? Cirugía del Trauma. Dpto. Cirugía Hospital de niños R. Gutiérrez, Bs As. 2002. [Fecha de acceso 15 de marzo de 2008]. Disponible en: http://www.paideianet. com.ar/mordeduras.htm

3.- Cofré R. Estudio epidemiológico de los accidentes por mordeduras de animales en la ciudad de Valdivia en el año. [Tesis pregrado MV]. Valdivia, Universidad Austral de Chile; 1995.

4.- Morgan M, Palmer J. Dog bites. BMJ 2007; 334: 413-7.

5.- Ministerio de Salud de Chile. Santiago: Departamento de Estadísticas de Información en Salud (DEIS); abril de 2007.

6.- Secretaría Regional Ministerial de Salud Región Valparaíso. Área Temática de Zoonosis. San Felipe: Oficina Provincial Aconcagua; abril de 2007.

7.- Ministerio de Salud de Chile. Santiago: Departamento de Estadísticas de Información en Salud (DEIS); septiembre de 2014.

8.- Talavera M. Simposio "Sobre población canina, un problema de salud pública"; Dirección General de Salud Ambiental, Ministerio de Salud, Gobierno del Perú; noviembre de 2013.

9.- Ministerio de Salud de la República Argentina. Gobierno de Mendoza: Subsecretaría de Planificación y Control, Departamento de Estadísticas; 158 [Fecha de acceso diciembre de 2014]. Disponible en: https://www.infosalud. mendoza.gov.ar/

10.- Lucero. E. Comunicación personal. Médico Veterinario. Etólogo Clínico. Centro Integral de Bienestar Animal, director programa de desarrollo cognitivo y socialización canina playgroupcanino.cl; Septiembre de 2015 y junio de 2016.

11.- Pageat P. Patología del comportamiento del perro. Ediciones pulso 2000; 326-42.

12.- Dehasse J. Todo sobre la psicología del gato. Editorial Servet 2008; 80-5.

13.- Ministerio de Salud de Chile. Normas Técnicas de Vigilancia de Enfermedades Transmisibles. División de Salud de las Personas, Departamento de Epidemiología. Región Metropolitana. [Fecha de acceso 15 de marzo de 2008]. Disponible en: http://epi.minsal.cl/epi/html/public/ enftransmisibles.pdf

14.- Reglamento de Prevención y Control de la Rabia en el Hombre y en los Animales. Diario Oficial de la República de Chile 29 de enero de 2014, cuerpo I-6, I-7 y I-8

15.- Ministerio de Salud de Chile. Norma General Técnica de Control y Prevención de Rabia en el Hombre y los Animales. Región Metropolitana. 2003. Disponible en: Anexo $\mathrm{N}^{\circ} 2$.

16.- Secretaría Regional Ministerial de Salud Región Metropolitana. Situación epidemiológica de Rabia en la Región Metropolitana. Santiago: Equipo de Zoonosis; 2006.

17.- Méndez P. Evaluación del programa nacional de prevención de la Rabia y sus componentes epidemiológicos en la comuna de la Granja 1990-1992. [Tesis Magíster en Salud Pública]. Santiago: Biblioteca Salud Pública, Facultad de Medicina. Universidad de Chile; 1995.

18.- Vergara Y. Epidemiología de las mordeduras por animales vertebrados, comuna de Puente Alto, período agosto de 1999-agosto de 2002. [Tesis pregrado MV]. Santiago, Universidad Iberoamericana de Ciencias y Tecnología; 1995.

19.- Aguayo M. Impacto de las mordeduras animales al hombre a través de protocolos de vacunación antirrábica. [Tesis pregrado MV]. Santiago: Biblioteca Medicina Veterinaria, Facultad de Ciencias Veterinarias y Pecuarias. Universidad de Chile. 1992.
20.- Instituto Nacional de Estadísticas. Resultados del Censo de Población y Vivienda. Santiago. V. I. 2002.

21.- Instituto Nacional de Estadísticas. Link solicitado a encargada SIAC, Región de Valparaíso Resultados del Censo de Población y Vivienda. Santiago. V. I. 2002. [Fecha de acceso julio de 2015]. Disponible en: http://www.ine.cl/canales/chile estadistico/ demografia_y_vitales/proyecciones2014/ proyecciones-de-poblacion-2014.xlsx

22.- Servicio de Salud Aconcagua: Red de atención primaria y secundaria en salud. [Fecha de acceso julio de 2015]. http://www. serviciodesaludaconcagua.cl

23.- Muñoz C. Estudio epidemiológico y significado económico de las mordeduras de perros ocurridas entre los años 1996 y 1998, en la ciudad de Valdivia. [Tesis pregrado MV]. Valdivia: Biblioteca Medicina Veterinaria, Facultad de Ciencias 156.

24.- Navia M J. Mordedura de perro en cara. Arch Argent Pediatr 2005; 103 (5): 396-400.

25.- Gómez J. Manual de Urgencias en Medicina Interna, Mordeduras por animales. Colombia. Ediciones Acta Médica Colombiana; 2002. [Fecha de acceso 15 de marzo de 2008]. Disponible en: http://www.aibarra.org/ guias/7-8.htm.

26.- Accidentes Traumatismos por Mordeduras. [Fecha de acceso 12 de abril de 2003]. Disponible en: www.buscamascotas.com.ar/ Pasteur folletos/mordeduras.htm.

27.- Méndez R, Gómez M, Somoza I, Liras J, Pais E, Vela D. Mordeduras de perro. Análisis de 654 casos en 10 años, La Coruña. 2002. [Fecha de acceso 12 de marzo de 2008]. Disponible en: http://db.doyma.es/cgi_bin/wdbcgi.exe/doyma/ mrevista.fulltext?pident $=13029673$.

28.- Kandaa K, Obayashia Y, Jayasingheb, Priyadarshania, Chandika D. Gamaged, Araia A, Tamashiroa H. Outcomes of a school-based 161 intervention on rabies prevention among school children in rural Sri Lanka. Int. Health first published online December 30, 2014 doi: 10.1093. Disponible en: http://inthealth. 
oxfordjournals.org/content/early/2014/12/30/ inthealth.ihu098.short.

29.- Bedregal P. "Ética de la investigación en Salud Publica" Rev. ARS Médica. 2006; 13 (13).

[Fecha de acceso enero de 2015]. Disponible en: http://escuela.med.puc.cl/publ/arsmedica/ ArsMedica13/IndiceArMedica13.html.

30.- Lolas F, Freitas G. Bioética. Santiago: Mediterráneo 2013; 10: 91.

31.- Pavletic C. Vigilancia de Rabia en Murciélagos insectívoros en Chile. Ministerio de Salud del Gobierno de Chile, Subsecretaria de Salud Pública. [Fecha de acceso 12 de julio de 2016].
Disponible en: http://www.paho.org/panaftosa/ index.php?option $=$ com_docman\&task $=\mathrm{doc}$ download\&gid $=415 \&$ Itemid $=311$.

32.- Villagra V. Caracterización epidemiológica de mordeduras en personas según registro de atención de urgencia Provincia de Los Andes, Chile (2005-2007). [Tesis Magíster en Salud Pública]. Santiago: Biblioteca Salud Pública, Facultad de Medicina. Universidad de Chile; 2015.

33.- Soto A. Análisis de un problema público no abordado el caso de los perros vagabundos y callejeros en Chile. [Tesis Magíster en Gestión y Políticas Públicas]. Santiago: Biblioteca Departamento de Ingeniería Industrial, Facultad de Ciencias Físicas y Matemáticas. Universidad de Chile; 2013.

34.- Control animal Rinconada de Los Andes. Ilustre Municipalidad de Rinconada. Departamento de Medio ambiente, 2014

35.- Wainnright I. Comunicación personal sobre personas mordidas notificadas desde los centros de salud Provincia de Los Andes 2013-2015. Encargado Unidad de Zoonosis, Oficina Provincial, Secretaría Regional Ministerial Aconcagua, marzo de 2017. 